\title{
Recognizing a plane-misoriented view of a familiar object is not influenced by the ease of specifying the main axis of elongation of that object
}

\author{
REBECCA LAWSON \\ University of Liverpool, Liverpool, England
}

\begin{abstract}
Participants saw three versions of pictures of familiar objects: the original unaltered (axis-normal) pictures, axis-extended pictures in which the main axes of the axis-normal pictures were elongated, and axis-switched pictures in which objects that were originally horizontally elongated were depicted as vertically elongated and vice versa. Relative to axis-normal pictures, axis extension aided decisions about whether the picture of the object was wide or tall, and axis switching hindered these decisions for both upright and plane-misoriented views. Nevertheless, although these axis manipulations clearly influenced decisions about the location of the object's main axis of elongation, axis-switched pictures were no harder to name than axis-extended pictures. Changing the depicted main axis of elongation by axis switching and axis extension did not influence object recognition in itself, whether for upright or for plane-misoriented views. This suggests that specifying the main axis of elongation of an object does not play an important role in the orientation-sensitive processes involved in identifying planemisoriented views of that object.
\end{abstract}

Plane-misoriented views of familiar objects are generally identified more slowly and less accurately than upright views of the same stimuli (Jolicœur, 1985). There is an ongoing debate as to the explanation for this misorientation cost and the mechanism by which a misoriented stimulus is identified. One common proposal is that an image transformation or normalization process is used to identify misoriented views, with this process usually taking longer to transform increasingly misoriented stimuli. Once the representation of an input stimulus has been transformed to an upright orientation, it can be matched to a stored upright object representation. No transformation is needed to identify upright views, since these can be matched directly to stored upright representations, so upright views can be identified more quickly. Recent evidence suggests that the image transformation process used is not mental rotation (Jolicœur, Corballis, \& Lawson, 1998; Lawson \& Jolicœur, 2003; see Lawson, 1999, for a review), but there is as yet no consensus as to what alternative transformation might be involved.

This research was supported by Grant RF\&G/2/9900393 from the Leverhulme Trust to the author. The article was written in part while I was on sabbatical at the Department of Psychology at the University of Otago, Dunedin, New Zealand. I thank the members of the department for their hospitality and generosity. Helpful comments were provided by Janice Murray and by the Visual Perception Lab Group at the University of Liverpool. Correspondence concerning this article should be sent to R. Lawson, Department of Psychology, University of Liverpool, Eleanor Rathbone Building, Bedford Street South, Liverpool L69 7ZA, England (e-mail: rlawson@ liverpool.ac.uk).

Note-This article was accepted by the previous editorial team, headed by Neil Macmillan.
Corballis (1988) noted an apparent paradox in this transformation account. For a misoriented stimulus to be transformed efficiently to its upright orientation, the transformation process would first need to determine the current orientation of the stimulus. Yet if the stimulus orientation was known, that would suggest that the stimulus had already been identified, in which case the transformation process would not be necessary. Corballis argued that this paradox could be resolved by assuming that participants usually successfully identify misoriented views of objects directly, without using a transformation process. Nevertheless, Corballis suggested that participants still sometimes double-check their initial identification by transforming representations of misoriented views to the upright and then matching them to stored upright representations. This double-checking would usually simply verify the accuracy of the initial orientation-insensitive identification. On this double-checking account, the misorientation cost of identification is due solely to the second stage of double-checking, and double-checking is usually unnecessary, because the object has normally already been successfully recognized. Evidence consistent with this account comes from studies suggesting that stimulus identity is usually verified more quickly than stimulus orientation for alphanumeric characters (Corballis, Zbrodoff, Shetzer, \& Butler, 1978) and line drawings of familiar objects (De Caro, 1998; De Caro \& Reeves, 2000, 2002).

However Jolicour and I have provided evidence against this double-checking account (Lawson \& Jolicœur, 1998, 1999). We used an identification task in which there should have been no disadvantage for double-checking, because 
only an unspeeded response was required (which would not penalize responses that involved double-checking). On the double-checking account, performance should have been orientation insensitive in this task. Contrary to this prediction, we found a strong misorientation cost. This indicates that the initial identification of objects is usually orientation sensitive.

In a proposal similar to the double-checking account, Hamm and McMullen (1998) argued that basic-level identification of objects is orientation invariant. They suggested that the usual finding of a disadvantage in naming plane-misoriented stimuli was due to some stimuli being identified at the subordinate level, with subordinatelevel identification being orientation sensitive. However Lawson and Jolicœur (2003) provided evidence against Hamm and McMullen's claims. They found that for most objects, identification at the basic level was orientation sensitive, as well as identification at the subordinate level (see also Murray, 1998).

Therefore, Corballis's (1988) paradox remains: How, without first identifying the object depicted, is the orientation of a misoriented picture determined so that a representation of that picture can be transformed efficiently to the upright? One hypothesis is that enough information may be available from a misoriented stimulus to determine its likely upright orientation but that this information is insufficient to fully identify the object. This information might include the main axes of elongation of an object (see Marr, 1982). These axes typically run horizontally or vertically through the object, due to the physical constraints of gravity. Other important clues to orientation might be the presence of symmetry or information common to a class of similarly shaped and similarly oriented stimuli (such as legs and wheels, which are typically found at the bottom of quadrupeds and wheeled vehicles, respectively; Jolicœur, 1990). If information such as the main axis of elongation reliably indicates the orientation of a stimulus, the appropriate transformation could be applied to the internal representation of a misoriented stimulus. This would transform the stimulus to the upright before it was identified. This two-stage account assumes that, first, the likely orientation of a stimulus is determined and, second, this information is then used to transform the internal representation of that stimulus to the upright so that it can be matched to stored object representations.

Priming evidence supporting such an account comes from Sanocki (1999), who reported that a line with the same orientation as the main axis of elongation of a target picture aided the identification of oblique targets even though the line cue contained no target-discriminating information. No such cuing was found for vertical targets. Sanocki argued that the latter result occurred because general cues to an environmental reference frame, such as gravity and the frame of the monitor, provided an effective vertical orientation cue for vertical targets. In addition, Gauthier and Tarr (1997) reported that recognition of a novel object could be improved by preceding it with a similarly shaped object shown at the same orientation. In contrast, evidence against this account comes from McMullen, Hamm, and Jolicœur (1995). They found that naming latencies were not reduced by either cuing the location of the top of a picture of a familiar object, using a dot, or cuing the orientation of the main top-bottom axis of the object, using an arrow.

In the four experiments reported here, I reexamined these issues. I focused on investigating the role of the main axis of elongation in the identification of misoriented views of familiar objects. If the main axis of elongation is used to specify object orientation and if it is important to determine the orientation of a misoriented picture of an object before it is recognized, misoriented pictures should be harder to identify when the main axis of elongation of the object is difficult to specify.

Evidence for the role of the axis of elongation in specifying object orientation comes from Sekuler and Swimmer (2000). They systematically manipulated the elongation and symmetry of line drawings of simple novel stimuli. Participants decided whether a horizontal or a vertical line would "most naturally go with" each stimulus. The participants responded using the axis of elongation, the axis of symmetry, or both if both were available. Furthermore, the importance of the axis of elongation in determining responses increased as the aspect ratio of the stimulus increased. Their results suggest that for their novel stimuli, the axis of elongation is important in determining the perceived orientation of that object, particularly for stimuli with large aspect ratios.

Sekuler and Swimmer's (2000) results suggest that the orientation of misoriented views could be specified by using a salient intrinsic cue to the main axis of the object; this information could then aid in the identification of the object. If so, elongation of the main axis of an object to make it more salient should improve the identification of misoriented views of that object. However, there are several limitations to the design of Sekuler and Swimmer's experiments that weaken this prediction. First, their participants decided only whether stimuli had horizontal or vertical axes; they did not have to identify the stimuli, so only the first part of the prediction was tested. Second, insofar as novel stimuli have any canonical orientation, Sekuler and Swimmer's stimuli (such as diamonds and ellipses) were either upright or $90^{\circ}$ plane rotated as a result of their symmetry and elongation. Their stimuli never appeared misoriented at oblique views. Third, Sekuler and Swimmer's stimuli were not depictions of familiar everyday objects, and their findings for simple novel two-dimensional objects might not generalize to such stimuli.

In the present experiments, I tested the hypothesis that if the main axis of elongation of a stimulus is easy (or difficult) to extract, this would specifically benefit (or disrupt) the identification of misoriented views of that stimulus. Axis-extended pictures manipulated the original axis-normal pictures of familiar objects in such a way as to accentuate the main axis of elongation by extend- 


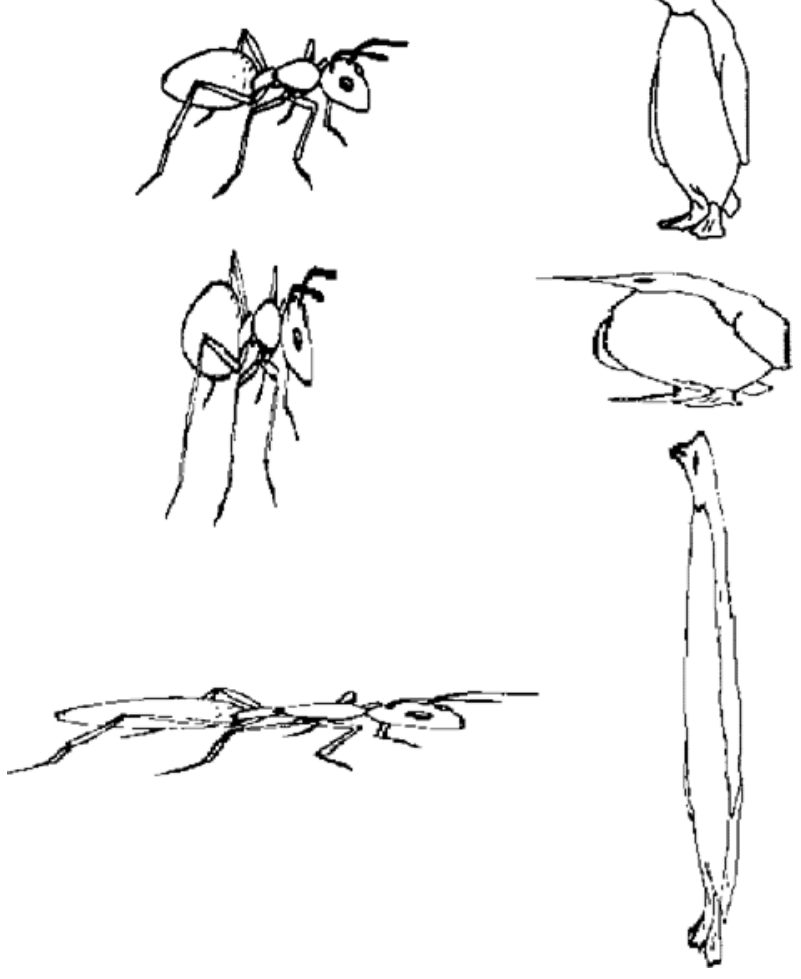

Figure 1. Examples of upright views of the experimental stimuli: At the top, unaltered axis-normal pictures; in the middle, axis-switched pictures; and at the bottom, axis-extended pictures, for a wide object (ant) on the left and for a tall object (penguin) on the right.

ing it, to make the axis more salient and easier to extract (see Figure 1). Axis-switched pictures were altered versions of the same axis-normal pictures, in which the position of the main axis of elongation was changed by switching its orientation by $90^{\circ}$. Axis-switched pictures had two perpendicular axes: the main axis of elongation of the picture itself and the usual main axis of the object depicted (see Figure 1). There were, therefore, two competing axes for axis-switched pictures, making it difficult to extract the main axis of elongation of the stimulus, whether for the picture itself or for the object depicted. Note that axis switching can be tested only for pictures of familiar objects that have a predefined axis of elongation, unlike the stimuli tested by Sekuler and Swimmer (2000).

Experiments 1 and 2 used a wide or tall stimulus? task analogous to Sekuler and Swimmer's (2000) horizontal or vertical axis? task. These experiments checked whether the orientation of the main axis of elongation of the object was easier to specify for axis-extended pictures and was harder to specify for axis-switched pictures, relative to the original axis-normal pictures of the objects. This prediction was confirmed for both upright stimuli and misoriented stimuli for participants who decided whether the pictures themselves were wide or tall. For participants who decided whether the object depicted was wide or tall, performance was again worse for both upright and misoriented views of axis-switched pictures, but there was no difference between axis-extended and axis-normal pictures.

In Experiments 3 and 4, the same axis-switched, axisextended, and axis-normal pictures as those in Experiments 1 and 2 were presented in an identification task. Experiment 3 directly tested the prediction that the misorientation cost for axis-switched pictures would be greater than that for axis-extended pictures given that, from the results of Experiments 1 and 2, the location of the main axis of elongation of axis-switched pictures was more difficult to specify. No evidence was found to support this prediction. Finally, Experiment 4 showed no evidence that the results of Experiment 3 were simply due to the high proportion of misoriented stimuli presented.

\section{EXPERIMENT 1}

This experiment was conducted to investigate whether the axis manipulations across the axis-extended, axisswitched, and axis-normal pictures would influence decisions about whether a stimulus was wide or tall. This experiment was necessary to demonstrate that these stimuli, which were also used in Experiments 3 and 4, were powerful enough to affect participants' ability to specify the main axis of elongation of the stimuli. If the axis manipulations did not affect these wide or tall decisions, they would not be expected to influence object identification in Experiments 3 and 4.

Dell'Acqua and colleagues (Dell' Acqua \& Job, 1998; Dell' Acqua, Job, \& Grainger, 2001) tested whether object identity influenced performance in a horizontal or vertical axis? task that did not require objects to be identified. They asked participants to specify the main axis of elongation of stimuli like the axis-normal and axisswitched pictures presented here. They found that for line drawings (but not for silhouettes), responses were faster to axis-normal than to axis-switched pictures of real familiar objects but that there was no difference between axis-normal and axis-switched pictures of meaningless nonobjects. The usual orientation of the main axis of elongation of a familiar object thus interfered with performance for the axis-switched line drawings. This interference occurred even though object identification was not required, since responses were made with respect to the picture itself, which was always upright.

Two groups of participants were tested in Experiment 1 . Both groups saw the same pictures of upright objects but did different tasks. The pictures group decided whether the main axis of elongation of a picture of a familiar object was wide (horizontal) or tall (vertical), as did the participants tested by Dell'Acqua and colleagues. The results from this group showed whether the axis manipulations directly influenced the participants' decisions about the location of the main axis of elongation of the picture. The objects group decided whether the main 
axis of elongation of the familiar object shown by the picture was wide or tall. The results from this group showed, first, whether people knew what the usual main axis of elongation for the objects depicted was and, second, whether performance was improved by making the axis of elongation more salient (for the axis-extended pictures) or was disrupted by altering the usual position of that axis (for the axis-switched pictures).

Axis elongation was expected to improve people's ability to specify the main axis of elongation of stimuli. If so, performance with axis-elongated pictures should be better than that with axis-normal pictures for both the pictures and the objects groups. In turn, for both groups, performance with axis-normal pictures should be better than that with axis-switched pictures. This was because, in contrast to the axis-normal and axis-extended pictures, for the axis-switched pictures the main axis of elongation of the picture and of the object depicted did not coincide. Instead, these two competing axes were perpendicular (see Figure 1).

\section{Method}

Participants. Sixteen undergraduate students at the University of Liverpool volunteered to take part in the experiment for course credit. Eight were assigned to the pictures group, and eight to the objects group. In this and the following experiments, all the participants were native speakers of English and had normal or correctedto-normal vision.

Materials. The original axis-normal pictures were 64 line drawings of familiar objects, most of which were taken from Snodgrass and Vanderwart (1980). The 64 objects were divided into four sets of 16 stimuli comprising the Tall-A and Tall-B sets (both of which had vertically elongated main axes in the upright view of the axisnormal picture; e.g., penguin) and the Wide-A and Wide-B sets (both of which had horizontally elongated main axes in the axisnormal upright picture; e.g., ant). ${ }^{1}$

Each tall and wide object was depicted in three versions: axis normal, axis extended and axis switched (see Figure 1). The axisnormal stimuli were the original pictures, which depicted the object with its usual canonical main axis of elongation. Axis-switched pictures were created by reversing the height-to-width ratio of an imaginary box that just enclosed the axis-normal picture. ${ }^{2}$ Thus, for the axis-normal picture of a penguin (a tall object), the enclosing imaginary box was around twice as high as it was wide, whereas the axisswitched penguin fitted into a box about two times wider than it was high. For axis-extended pictures, the height-to-width ratio of the imaginary enclosing box around the original axis-normal picture was made more extreme. The length of the longer side of this imaginary box was increased to 384 pixels, and the length of the shorter side was reduced until the area of the imaginary box that just enclosed the axis-extended picture equaled the area enclosing the axis-normal picture. Thus, the imaginary boxes that just enclosed the axis-switched, axis-extended, and axis-normal pictures of a given object all had the same area, with only the width and the height of the box changing across the stimuli.

The average ratio of the sides of the imaginary box was $1.6: 1$ for both the axis-normal and the axis-switched pictures, and it was 4.9:1 for the axis-extended pictures. The effect of axis switching and axis extension varied across different objects, depending on the height and width of the imaginary box that just enclosed the axisnormal picture. I was interested in the qualitative effects of axis switching and axis extension. It would be diff icult to provide a meaningful quantitative measure of the perceptual effects of these axis manipulations. The dimensions of the smallest enclosing box around a given picture provides only a crude indication of the elongation of the main axis of that picture. In addition, the effectiveness of axis switching and axis extension will depend on the variability in the main axis of different exemplars of that object in the world. There is more variability in the relative height-to-width ratio of trees than of pineapples, so there may be less effect from axis manipulations of trees than of pineapples.

Upright views of the axis-normal, axis-switched and, axis-extended pictures of each object were produced that depicted the object at its environmentally predominant orientation. Each stimulus was also plane rotated by $120^{\circ}$ for use in Experiments 2 and 3. A rotation of $120^{\circ}$ was used because this usually produces the maximum misorientation cost on recognition (Jolicœur, 1990).

Design. The participants completed one experimental block of 192 trials. In this block, there were 3 trials that presented pictures of each of the 64 objects. These 3 trials presented upright views of the axis-normal, axis-extended, and axis-switched pictures of the object. The trials were presented in a different random order for each participant. Prior to the experimental block, the participants completed a block of 29 practice trials in which upright views of axis-normal, axis-extended, and axis-switched pictures were presented. No stimuli seen in practice were presented in the experimental block.

Apparatus and Procedure. A Macintosh 7600/132 computer running the PsyScope Version 1.0.2b experimental presentation software was used to display the stimuli. The experiment lasted around $20 \mathrm{~min}$. The procedure for each trial was as follows. A central fixation cross appeared on the screen for $400 \mathrm{msec}$, followed after $100 \mathrm{msec}$ by a picture of an object. This was displayed centrally until the participant responded by making either a $\mathrm{W}$ or a $\mathrm{T}$ keypress. After $300 \mathrm{msec}$, "W-wide" or "T-tall" was presented centrally as written feedback for $1,000 \mathrm{msec}$. There was an intertrial interval of $1,000 \mathrm{msec}$. There was a self-timed break between the practice and the experimental blocks. The participants were told to make their responses as rapidly and accurately as possible, and they were warned that some objects would look distorted.

The pictures group was told to respond "T," for tall, if the picture was vertically elongated about its main axis (e.g., an axis-normal or axis-extended picture of a penguin or an axis-switched picture of an ant), regardless of the elongation of the object in the world. They were told to respond "W," for wide, if the picture was horizontally elongated about its main axis (e.g., an axis-normal or axis-extended picture of an ant or an axis-switched picture of a penguin), regardless of the elongation of the object in the real world. The objects group was instructed to respond "T," for tall, if the real-world object depicted was vertically elongated (e.g., penguin) and "W," for wide, if it was horizontally elongated (e.g., ant), regardless of whether an axis-normal, axis-extended, or axis-switched picture was presented.

\section{Results}

Trials with reaction times (RTs) less than $500 \mathrm{msec}$ or greater than 2,500 msec were discarded as errors (less than $2 \%$ of the trials). No participants were replaced. Analyses of variance (ANOVAs) were conducted on the mean correct RTs and percentages of errors. In this and the following experiments, the results for by-participants and by-items analyses are reported by using subscripts $F_{\mathrm{p}}$ and $F_{\mathrm{i}}$, respectively. There were three within-subjects factors (if-distorted [axis-normal, axis-switched, or axisextended], three-dimensional [3-D] object axis [wide or tall], and stimulus set [Set A or Set B]) and one betweensubjects factor (task [objects or pictures decision]). The 3-D object axis and stimulus set counterbalancing factors were included in the analyses, but effects involving 
these factors were not the focus of interest, and so, for clarity of presentation, they are not reported here. All comparisons noted are significant at $p<.05$ in post hoc Newman-Keuls analyses.

The wide or tall decision for upright views only. If-distorted was significant, whereas task was significant for items only for RTs. These two main effects were qualified by a significant interaction of if-distorted and task for both RTs $\left[F_{\mathrm{p}}(2,28)=14.634, p<.001 ; F_{\mathrm{i}}(2,120)=\right.$ $31.408, p<.001$; see Figure 2] and errors $\left[F_{\mathrm{p}}(2,28)=\right.$ $\left.4.184, p<.03 ; F_{\mathrm{i}}(2,120)=3.400, p<.04\right]$. For RTs, for the pictures group, axis-extended pictures $(688 \mathrm{msec})$ were classified most quickly as wide or tall. Axis-normal pictures $(861 \mathrm{msec})$ were classified more slowly, and axis-switched pictures $(1,037 \mathrm{msec})$ were classified most slowly. In contrast, for the objects group, axis-extended pictures $(917 \mathrm{msec})$ and axis-normal pictures $(907 \mathrm{msec})$ were classified equally quickly, although again axisswitched pictures $(1,044 \mathrm{msec})$ were classified most slowly. For errors, for both groups, axis-extended pictures $(1.4 \%$ and $5.5 \%$ for the pictures and the objects groups, respectively) and axis-normal pictures ( $7.6 \%$ for both groups) were classified equally accurately, whereas axis-switched pictures $(27.9 \%$ and $21.9 \%$ for the pictures and the objects groups, respectively) were classified less accurately. In addition, for the axis-switched pictures only, the pictures group was less accurate than the objects group. If-distorted, therefore, had significantly more influence on the pictures group than on the objects group for both RTs and errors.

\section{Discussion}

Altering the main axis of elongation of a picture of an object strongly influenced decisions about whether the

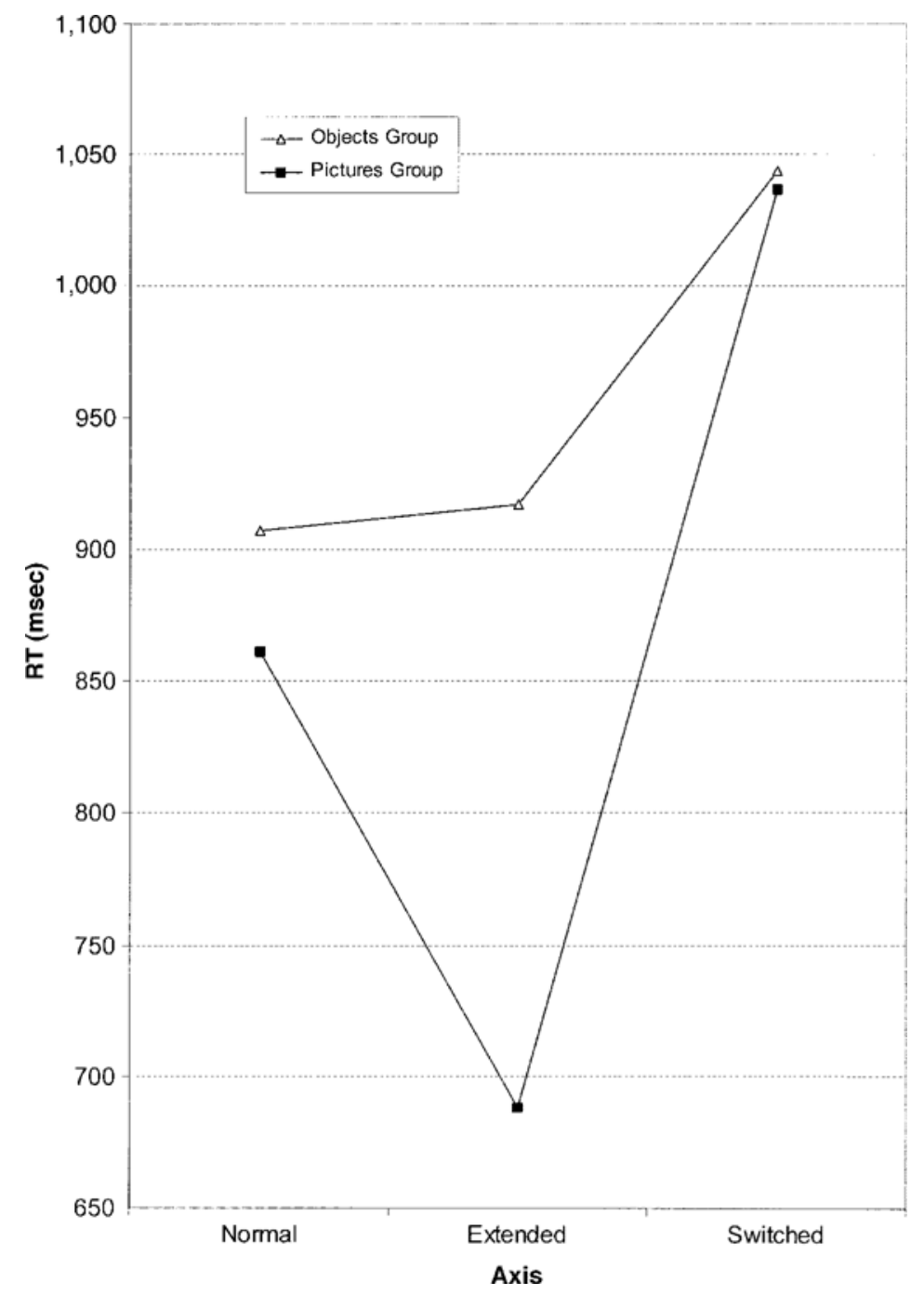

Figure 2. Mean reaction times (RTs) to decide whether a stimulus was wide or tall for upright axis-normal, axis-extended, and axis-switched pictures in Experiment 1. 
picture itself was wide or tall (for the pictures group) and whether the object depicted was wide or tall (for the objects group; see Figure 2). For both groups, decisions were easier for undistorted axis-normal pictures than for axis-switched pictures, in which the main axis of elongation of the picture was perpendicular to the usual main axis of elongation of the object depicted. In addition, for the pictures group only, decisions were easier for axisextended pictures, in which the usual main axis of the object was elongated, than for axis-normal pictures.

The pictures group replicated the finding of Dell'Acqua and Job (1998) that it was easier to classify axis-normal pictures as wide or tall, as compared with axis-switched pictures. Furthermore, this result for the pictures group was extended by finding that it was easier to classify axis-extended pictures as wide or tall than axis-normal pictures. The good overall performance of the objects group demonstrated that the participants knew the usual orientation of the main axis of elongation of the familiar objects tested. Their performance was, though, worse for axis-switched than for axis-normal and axis-extended pictures. The objects group was thus sensitive to the main axis of elongation of the picture itself, and this interfered with their decisions to the axis-switched pictures.

The axis manipulations had more influence on the pictures group than on the objects group. The objects group only had to use the picture presented to try to identify the object before they retrieved the location of the object's main axis of elongation from memory. Indeed for the objects group, responding to the depicted axis manipulations would have led to errors for the axis-switched pictures. In contrast, for the pictures group, the main axis of elongation of the picture itself needed to be extracted, so the salience of that axis was more critical to performance for this group. For the objects group, the increased ease of specifying the main axis of elongation of axisextended pictures was probably offset by the increased difficulty in identifying those pictures, relative to undistorted axis-normal pictures, resulting in similar levels of performance for axis-extended and axis-normal pictures. This was not the case for the pictures group. Specifying the location of the main axis of elongation of the picture was important for the pictures, but not for the objects, group, whereas identifying the stimulus was unnecessary for the pictures, but not for the objects, group. This then explains why axis manipulations had more effect on the performance of the pictures group.

\section{EXPERIMENT 2}

Experiment 1 revealed that the axis manipulations across axis-extended, axis-switched, and axis-normal pictures had a powerful influence on decisions about the location of the main axis of elongation of a stimulus. However, in Experiment 1, as in Dell'Acqua and Job (1998), only upright stimuli were presented. Experiment 2 tested whether the axis manipulations had the same effect on classifying misoriented, as well as up- right, views of objects as wide or tall. As in Experiment 1, Experiment 2 tested a pictures group who decided whether the main axis of elongation of the picture itself was wide or tall and an objects group who decided whether the main axis of elongation of the object represented by the picture was wide or tall. The stimuli in Experiment 2 were seen both in their usual upright orientations in the plane and $120^{\circ}$ plane misoriented. The pictures group was instructed to use wide or tall responses to describe the main axis of elongation of the stimulus as if the stimulus were shown upright. Therefore, the inclusion of misoriented stimuli forced the pictures group, as well as the objects group, to identify the stimuli, at least coarsely.

If, when identifying misoriented views, it is important to find an object's main axis of elongation in order to indicate the likely orientation of the object, any benefit for axis-extended over axis-normal pictures and any benefit for axis-normal over axis-switched pictures should be greater for misoriented than for upright views. According to this account, the misorientation cost was predicted to be particularly sensitive to axis manipulations. It was expected to be smallest for axis-extended pictures (where the main axis of elongation was highly salient) and largest for axis-switched pictures (where the main axes of elongation of the picture and of the object depicted were perpendicular).

\section{Method}

Participants. Twenty-four undergraduate students at the University of Liverpool volunteered to take part in the experiment for course credit. Twelve were assigned to the pictures group, and 12 to the objects group.

Materials. Upright and misoriented views of the axis-normal, axis-switched, and axis-extended pictures of the 16 Tall-A, 16 Tall-B, 16 Wide-A, and 16 Wide-B objects presented in Experiment 1 were used here.

Design. The participants completed one experimental block of 384 trials. In this block, there were 6 trials that presented pictures of each of the 64 objects. These 6 trials presented an upright and a misoriented view of the axis-normal, axis-extended, and axis-switched pictures of the object. The trials were presented in a different random order for each participant. Prior to the experimental block, the participants completed a block of 38 practice trials in which upright and misoriented views of axis-normal, axis-extended, and axisswitched objects were presented. No objects seen in practice were presented in the experimental trials.

Apparatus and Procedure. The apparatus and procedure were identical to those in Experiment 1, except that the experiment lasted around $35 \mathrm{~min}$ and the participants were warned that some objects would be depicted at unusual angles and that they should decide whether the stimulus was wide or tall with respect to the canonical upright orientation of the object.

\section{Results}

Trials with RTs less than $400 \mathrm{msec}$ or more than $2,500 \mathrm{msec}$ were discarded as errors (less than $1 \%$ of the trials). No participants were replaced. ANOVAs were conducted on the mean correct RTs and the percentages of errors. Three sets of analyses were conducted for responses to upright views only, misoriented views only, and the misorientation cost (the difference between mis- 
oriented and upright views). In all analyses, there were three within-subjects factors, if-distorted (axis-normal, axis-switched, or axis-extended), 3-D object axis (wide or tall), and stimulus set (Set A or Set B), and one betweensubjects factor, task (objects or pictures decision). The 3-D object axis and stimulus set counterbalancing factors were included in the analyses, but effects involving these factors were not the focus of interest, and so, for clarity of presentation, they are not reported here. All comparisons noted are significant at $p<.05$ in post hoc Newman-Keuls analyses.

The wide or tall decision for upright views. Ifdistorted was significant, whereas task was significant only for items. These two main effects were qualified by a significant interaction of if-distorted and task for both RTs $\left[F_{\mathrm{p}}(2,44)=22.657, p<.001 ; F_{\mathrm{i}}(2,120)=27.806\right.$, $p<.001$; see Figure 3$]$ and errors $\left[F_{\mathrm{p}}(2,44)=14.351\right.$, $\left.p<.001 ; F_{\mathrm{i}}(2,120)=18.350, p<.001\right]$. For the pictures group, axis-extended pictures (719 msec, $2.5 \%$ ) were easiest to classify as wide or tall. Axis-normal pictures (845 msec, $10.0 \%$ ) were harder to classify (not significant for participants for errors). Axis-switched pictures $(1,022 \mathrm{msec}, 31.6 \%)$ were hardest to classify. In contrast, for the objects group, axis-extended pictures $(732 \mathrm{msec}$, $7.6 \%)$ and axis-normal pictures $(736 \mathrm{msec}, 6.9 \%)$ were classified equally well, although again axis-switched pictures ( $834 \mathrm{msec}, 14.8 \%$ ) were harder to classify. For upright views, if-distorted therefore had significantly more influence on the pictures group than on the objects group.

The wide or tall decision for misoriented views. The results for misoriented views were very similar to those for upright views. If-distorted and task were both significant. These two main effects were qualified by a significant interaction of if-distorted and task for both RTs $\left[F_{\mathrm{p}}(2,44)=10.804, p<.001 ; F_{\mathrm{i}}(2,120)=19.833, p<\right.$ .001 ; see Figure 3$]$ and errors $\left[F_{\mathrm{p}}(2,44)=19.895, p<\right.$

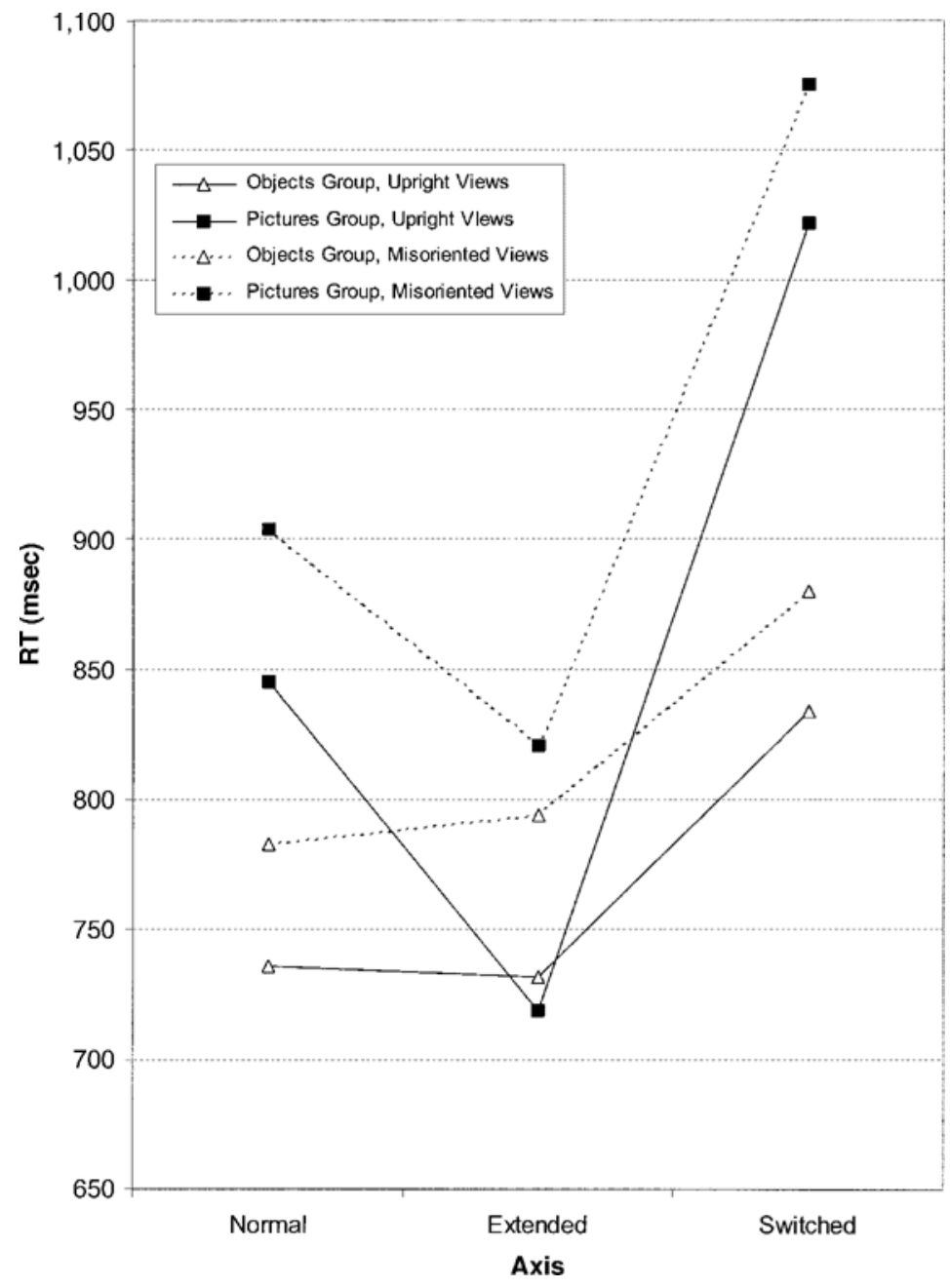

Figure 3. Mean reaction times (RTs) to decide whether a stimulus was wide or tall for upright and misoriented axis-normal, axis-extended, and axisswitched pictures in Experiment 2. 
$\left..001 ; F_{\mathrm{i}}(2,120)=9.507, p<.001\right]$. For the pictures group, axis-extended pictures $(821 \mathrm{msec}, 3.9 \%)$ were easiest to classify as wide or tall. Axis-normal pictures $(904 \mathrm{msec}$, $14.2 \%$ ) were harder to classify (not significant for participants for errors). Axis-switched pictures $(1,075 \mathrm{msec}$, $29.7 \%$ ) were hardest to classify. In contrast, for the objects group, axis-extended pictures (794 msec, 6.9\%) and axisnormal pictures ( $783 \mathrm{msec}, 6.9 \%)$ were classified equally well, although again axis-switched pictures $(880 \mathrm{msec}$, $16.3 \%$ ) were harder to classify. For misoriented views, if-distorted therefore had significantly more influence on the pictures group than on the objects group.

The misorientation cost on the wide or tall decision. The misorientation cost was calculated as the increase in RTs or errors to misoriented $120^{\circ}$ pictures, as compared with upright $0^{\circ}$ pictures. Overall, there was a misorientation cost of $62 \mathrm{msec}$ on RTs and $0.7 \%$ on errors. A final set of ANOVAs analogous to those conducted above were conducted on the misorientation cost. There were no significant effects in the RT analyses and no reliably significant effects in the error analyses. For the pictures group, the misorientation cost was no less for axisextended pictures $(102 \mathrm{msec}, 1.4 \%)$ than for axis-normal pictures (59 msec, $4.2 \%$ ), for which, in turn, the misorientation cost was no less than that for axis-switched pictures (53 msec, $-2.0 \%)$. Likewise, for the objects group, the misorientation cost was no less for axis-extended pictures $(63 \mathrm{msec},-0.7 \%)$ than for axis-normal pictures (47 msec, $0.0 \%$ ), for which, in turn, the misorientation cost was no less than that for axis-switched pictures $(46 \mathrm{msec},-1.4 \%)$. These results do not support the predictions made on the basis that the location and salience of the main axis of elongation of a stimulus specifically influences the identification of misoriented views of objects. Instead, these results suggest that axis position and extent does not affect the recognition of misoriented stimuli.

\section{Discussion}

As in Experiment 1, the depiction of an object's main axis of elongation had a strong and systematic influence on the participants' ability to classify as wide or tall either the picture itself (for the pictures group) or the object depicted (for the objects group; see Figure 3). For both groups, the pattern of performance was the same for upright and plane-misoriented views. Replicating the results of Experiment 1, the pictures group found the axisextended pictures easiest to classify, the axis-normal pictures harder to classify, and the axis-switched pictures hardest to classify. Furthermore, the pictures group results extended the findings of Experiment 1 to misoriented views and confirmed that the axis manipulations tested here had a powerful influence on the participants' ability to specify the main axis of elongation of a stimulus. The objects group found the axis-extended and axisnormal pictures equally easy to classify as wide or tall, although like the pictures group, they found the axisswitched pictures harder to classify. These objects group results replicated Experiment 1 and extended the findings to misoriented views. For both groups, performance was impaired if there was a conflict between the main axes of elongation of the picture and of the object shown in the picture, as was the case for the axis-switched stimuli. However, as in Experiment 1, the objects group was less sensitive to axis manipulations than was the pictures group.

Importantly, for neither group was the misorientation cost for axis-extended pictures less than that for axisnormal pictures, and this misorientation cost was, in turn, no less than that for axis-switched pictures. This suggests that the main axis of elongation of a picture is not an important cue to the orientation of the picture that can influence the identification of misoriented pictures (cf. Sekuler \& Swimmer, 2000). However, Experiment 2 tested only wide or tall decisions about the main axis of elongation. The effects of the axis-switching and axisextending manipulations on object recognition were not tested directly with an identification task. To address this, Experiment 3 was performed to examine whether the misorientation cost for naming would be larger for axis-switched pictures than for axis-extended pictures.

\section{EXPERIMENT 3}

The most important result from Experiments 1 and 2 was that it was much easier to decide whether stimuli were wide or tall for axis-extended pictures than for axisswitched pictures (see Figures 2 and 3). This finding indicates that these axis manipulations were strong enough to influence decisions about the orientation of the main axis of elongation of stimuli. In addition, in Experiment 2 , the misorientation cost was no smaller for axisextended than for axis-switched pictures. This finding fails to support the prediction that the location of the main axis of elongation of a misoriented picture can be used to indicate the orientation of the picture and that this information, in turn, can help to identify the misoriented picture by guiding orientation-sensitive transformation processes. This account would predict a smaller misorientation cost for the axis-extended pictures than for the axis-switched pictures since, from the results of Experiments 1 and 2, the main axis of elongation was easier to specify for the axis-extended pictures.

However, in Experiment 2, specifying the main axis of elongation of the stimulus was the primary task. Object identification was just one component of that task. In Experiment 2, both the pictures and the objects groups did have to identify the objects depicted in order to classify stimuli as wide or tall. For the pictures group, this was because participants were instructed that responses were to be made with respect to whether the picture would be wide or tall if it were shown upright. For the objects group, this was because participants were instructed that responses were to be made with respect to the main axis of elongation of the familiar object that was depicted. Nevertheless, in Experiment 2, the potential for axis manipulations to influence the misorienta- 
tion cost, due to their effects on object identification, may have been small, particularly for the pictures group. In contrast, in Experiment 3, the primary task was object identification, so the experiment should be more sensitive to any effects of axis manipulations on the misorientation cost. Experiment 3 tested directly whether axis manipulations specifically influenced object identification and, in particular, whether the misorientation cost for axis-extended pictures was less than that for axisswitched pictures.

Studies have shown that verifying stimulus identity is faster than verifying stimulus plane orientation (Corballis et al., 1978; De Caro, 1998; De Caro \& Reeves, 2000, 2002). This finding could be taken to indicate that object orientation is determined only after object identity is known. If so, axis manipulations that vary the ease of determining object orientation could not influence the identification of misoriented objects. However, the time taken to verify stimulus orientation might not reflect the time course of any of the putative processes used to determine object orientation in order to identify misoriented objects. The output of the latter processes might not be available to the decision-making processes involved in explicitly verifying stimulus orientation. Object recognition processes in the visual system might determine object orientation before object identity even if people's explicit decisions about object identity are faster than their decisions about object orientation.

Misorientation costs to name axis-normal, axisextended, and axis-switched pictures of objects were compared in Experiment 3. The main axis of elongation of the picture coincided with the main axis of elongation of the object depicted for the axis-normal and axisextended pictures, but it was perpendicular to the main axis of the object depicted for the axis-switched pictures. The results of Experiments 1 and 2 indicated that it was harder to specify the orientation of the main axis of elongation of axis-switched pictures than of axis-normal or axis-elongated pictures. This was the case both when wide or tall decisions were made about the picture itself (for the pictures groups) and when these decisions were made about the object depicted (for the objects groups). If, first, the main axis of elongation plays an important role in specifying the orientation of misoriented stimuli and, second, efficient recognition requires the orientation of misoriented stimuli to be known, the misorientation cost for axis-switched pictures should be greater than that for axis-normal and axis-extended pictures.

However, any increase in the misorientation cost for the axis-switched pictures relative to the axis-normal pictures could simply be due to the axis-switched pictures being harder to identify in general, rather than being due to a specific effect related to the main axis of elongation. Image information such as global outline shape and fine detail was altered or lost for the axisswitched pictures, relative to the axis-normal pictures. For this reason, even upright axis-switched pictures were expected to be harder to name than upright axis-normal pictures. Furthermore, misorientation costs can be larger for stimuli that are more difficult to identify (e.g., Lawson \& Jolicœur, 1998, 2003). The axis-extended pictures were necessary to test this alternative hypothesis. Both axis extension and axis switching were expected to disrupt the identification of upright views of the objects. If this disruption was simply due to these axis manipulations' degrading the image (with the different effects of the two manipulations on the main axis of elongation being irrelevant), a similar, large misorientation cost would be predicted for both axis-switched and axisextended pictures, relative to axis-normal pictures. In contrast, if upright views of both axis-switched and axisextended pictures were harder to name but, in addition, the misorientation cost was greater for axis-switched than for axis-extended pictures, this would suggest that the nature of the axis manipulation itself was important when misoriented views were identified.

\section{Method}

Participants. Sixty-four undergraduate students at the University of Liverpool volunteered to take part in the experiment for course credit.

Materials. Upright and misoriented views of the axis-normal, axis-switched, and axis-extended pictures of the 16 Tall-A, 16 Tall-B, 16 Wide-A, and 16 Wide-B objects presented in Experiment 1 were used here.

Design. The participants completed three experimental blocks of naming. There were 64 experimental trials per block. The participants named each of the 64 objects on 1 trial in every block. For each of the four sets of objects (16 Tall-A, 16 Tall-B, 16 Wide-A, and 16 Wide-B), 8 objects were seen upright, and 8 were seen misoriented, in each block. For a given participant, the subset of 8 objects presented at a given view (upright or misoriented) in one block were presented at the other view in the subsequent block, whereas the same version of the object (axis normal, axis switched, or axis extended) was presented in every block. The first and third blocks were, therefore, identical for a given participant, except that the trials were presented in a different random order in every block.

Eight participants were assigned to each of eight subgroups, which differed on whether they saw the tall-group or the widegroup stimuli, whether Set A or B stimuli were distorted, and which subset of stimuli were presented upright in Block 1. The participants presented with the tall-group stimuli saw axis-normal Tall-A (or B) and axis-normal Wide-A (or B) stimuli, axis-extended Tall-B (or A) stimuli, and axis-switched Wide-B (or A) stimuli. Those shown the wide group stimuli saw axis-normal Tall-A (or B) and axis-normal Wide-A (or B) stimuli, axis-extended Wide-B (or A) stimuli, and axis-switched Tall-B (or A) stimuli. Prior to the first block of naming, the participants completed a block of 16 practice trials, half showing upright views and half showing misoriented views. Practice trials were identical to experimental trials, except that no axis-normal pictures were presented; instead, a mixture of axis-extended and axis-switched pictures were presented to increase the participants' exposure to these distortions. No objects seen in practice were presented in the experimental trials.

Apparatus and Procedure. A Macintosh 7600/132 computer running the PsyScope Version 1.0.2b experimental presentation software was used to display the stimuli. The experiment lasted about $20 \mathrm{~min}$.

The procedure for each trial was as follows. A central fixation cross appeared on the screen for $500 \mathrm{msec}$, immediately followed by a picture of an object. This was displayed centrally until the participant responded by naming the object aloud. RTs were recorded 
by the computer using a microphone and a voice-activated relay. The name of the object was then presented centrally as written feedback until the experimenter coded the accuracy of the participant's response. There was an intertrial interval of $750 \mathrm{msec}$. There was a self-timed break between each block.

The participants were told to name the objects as rapidly and accurately as possible. They were warned that some objects would look distorted and that some objects would be depicted at unusual angles, and they were told to ignore these manipulations.

\section{Results}

Trials in which the participants used an inappropriate name or in which the microphone was accidentally activated before the participant had responded were discarded as errors. In addition, RTs less than $500 \mathrm{msec}$ or exceeding 2,500 msec were discarded as errors (less than $2 \%$ of the trials). The participants were replaced if they scored over $17.5 \%$ errors. Three wide-group participants and 1 tall-group participant were replaced using this criterion. ANOVAs were conducted on the mean correct RTs and the percentages of errors. Two sets of analyses were conducted. In the first set, the dependent variable was the misorientation cost, calculated as the increase in RTs or errors when misoriented $120^{\circ}$ pictures were named, as compared with upright $0^{\circ}$ pictures. These analyses investigated the influence of the main axis of elongation on naming misoriented views over and above any axis manipulation effects on naming upright views. These were the most theoretically important analyses. In the second set of analyses, the dependent variable was the speed or the accuracy of naming the upright views only.

In all analyses, there were three within-subjects factors, if-distorted (unaltered, axis normal; distorted, either axis extended or axis switched), type-distortion (for the distorted pictures, either axis extended or axis switched; this was a dummy variable for the axis-normal pictures), and block $(1,2$, or 3$)$, and two between-subjects factors, group (tall-group or wide-group stimuli) and stimulus set (if Set A was presented unaltered and Set B distorted or vice versa). Interactions involving the group counterbalancing factor were not the focus of interest, and so, for clarity of presentation, they are not reported here. All comparisons noted are significant at $p<.05$ in post hoc Newman-Keuls analyses.

The misorientation cost on naming. If-distorted was significant for RTs $\left[F_{\mathrm{p}}(1,60)=23.698, p<.001\right.$; $\left.F_{\mathrm{i}}(1,60)=56.760, p<.001\right]$ and errors $\left[F_{\mathrm{p}}(1,60)=\right.$ $\left.59.198, p<.001 ; F_{\mathrm{i}}(1,60)=32.202, p<.001\right]$. The misorientation cost was larger for distorted (axisswitched and axis-extended) pictures (114 msec, 7.0\%) than for axis-normal pictures (51 msec, $0.5 \%)$.

Block was significant for RTs $\left[F_{\mathrm{p}}(2,120)=4.341, p<\right.$ $\left..02 ; F_{\mathrm{i}}(2,120)=6.590, p<.002\right]$ and errors $\left[F_{\mathrm{p}}(2,120)=\right.$ $\left.18.534, p<.001 ; F_{\mathrm{i}}(2,120)=18.824, p<.001\right]$. The misorientation cost was reduced from Block $1(107 \mathrm{msec}$, $7.7 \%$ ) to Blocks 2 (68 msec, $1.4 \%$ ) and 3 (73 msec, $2.1 \%$ ). In addition, the interaction of block and if-distorted was significant for errors only $\left[F_{\mathrm{p}}(2,120)=15.425, p<.001\right.$; $\left.F_{\mathrm{i}}(2,120)=16.453, p<.001\right]$. The reduction in the mis- orientation cost on errors with practice was greater for distorted than for axis-normal pictures.

Most important, the interaction of if-distorted and type-distortion was not significant for RTs $\left[F_{\mathrm{p}}(1,60)=\right.$ $0.283, p>.5 ; F_{\mathrm{i}}(1,60)=0.105, p>.7$; see Figure 4$]$ or errors $\left[F_{\mathrm{p}}(1,60)=0.070, p>.7 ; F_{\mathrm{i}}(1,60)=0.051\right.$, $p>$.8]. In particular, the misorientation cost was similar for both types of distorted stimuli: axis-extended pictures (113 msec, 6.9\%) and axis-switched pictures (115 msec, $7.1 \%)$.

Naming upright views. If-distorted was significant for RTs $\left[F_{\mathrm{p}}(1,60)=89.815, p<.001 ; F_{\mathrm{i}}(1,60)=74.551\right.$, $p<.001]$ and errors $\left[F_{\mathrm{p}}(1,60)=19.966, p<.001\right.$; $\left.F_{\mathrm{i}}(1,60)=14.036, p<.001\right]$. Distorted (axis-switched and axis-extended) pictures were harder to name $(960 \mathrm{msec}$, $7.4 \%$ ) than axis-normal pictures (884 $\mathrm{msec}, 4.5 \%)$.

Block was significant for RTs $\left[F_{\mathrm{p}}(2,120)=90.887\right.$, $\left.p<.001 ; F_{\mathrm{i}}(2,120)=66.108, p<.001\right]$ and errors $\left[F_{\mathrm{p}}(2,120)=69.825, p<.001 ; F_{\mathrm{i}}(2,120)=31.497, p<\right.$ $.001]$. Practice improved performance from Block 1 (995 msec, $10.7 \%$ ) to Block 2 (920 msec, $4.1 \%$ ) and then to Block 3 ( $851 \mathrm{msec}, 3.0 \%$; the latter difference was not significant for errors). In addition, the interaction of block and if-distorted was significant for RTs for items $\left[F_{\mathrm{p}}(2,120)=1.411, p>.2 ; F_{\mathrm{i}}(2,120)=4.768, p<.02\right]$ and was significant for errors $\left[F_{\mathrm{p}}(2,120)=5.924, p<\right.$ $\left..004 ; F_{\mathrm{i}}(2,120)=4.479, p<.02\right]$. The improvement in naming with practice was greater for distorted than for axis-normal pictures.

Most important, the interaction of if-distorted and type-distortion was not significant for RTs $\left[F_{\mathrm{p}}(1,60)=\right.$ $0.105, p>.7 ; F_{\mathrm{i}}(1,60)=0.015, p>.9$; see Figure 4$]$ or errors $\left[F_{\mathrm{p}}(1,60)=1.967, p>.1 ; F_{\mathrm{i}}(1,60)=1.135, p>.2\right]$. In particular, both types of distorted stimuli were equally difficult to name: axis-extended pictures $(962 \mathrm{msec}$, $6.6 \%$ ) and axis-switched pictures (957 msec, 8.1\%).

\section{Discussion}

Misoriented views were harder to name than upright views throughout Experiment 3. This misorientation cost was reduced with practice, replicating many other studies (Jolicœur, 1985; Jolicœur \& Milliken, 1989; Lawson, 1999). However even in the third block, misoriented views were still harder to name than upright views. This indicates that throughout Experiment 3, objects were being identified using orientation-sensitive recognition processes.

Experiment 3 investigated whether these orientationsensitive processes use an object's main axis of elongation to determine its orientation. Knowledge of object orientation could, in turn, guide transformation processes used to identify misoriented views of an object. For axisswitched pictures, the usual orientation of the main axis of elongation of a given familiar object was depicted as horizontal, rather than vertical, or vice versa. Thus, it was likely that the incorrect stimulus orientation would be assigned to axis-switched pictures. This should be particularly disruptive when misoriented, as compared with upright, views of axis-switched pictures were identified. 


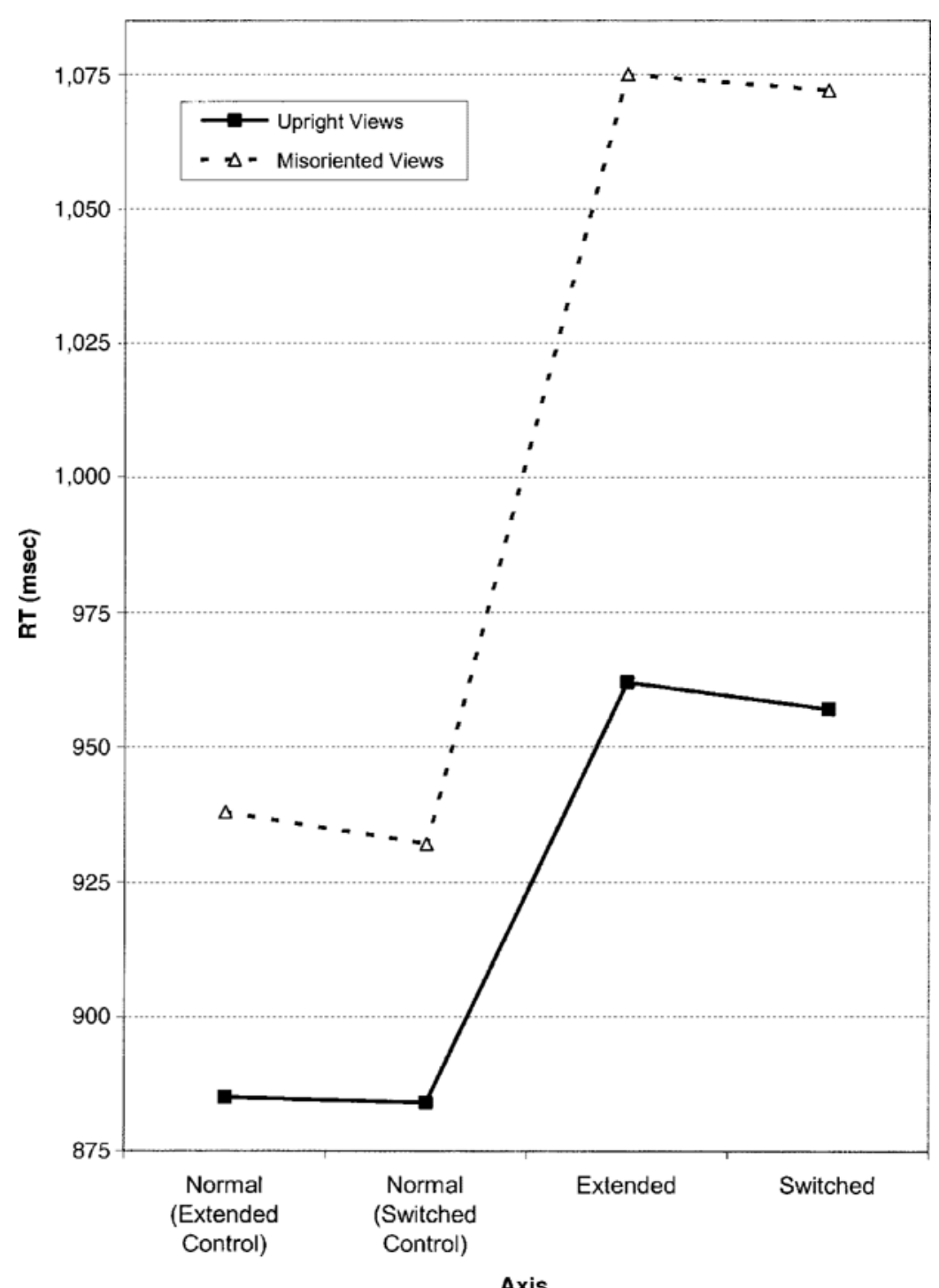

Figure 4. Mean reaction times (RTs) to name upright and misoriented axis-normal, axis-extended, and axis-switched pictures in Experiment 3.

Upright views would not need to be transformed using orientation-sensitive processes prior to identification, since they could be matched directly to stored upright object representations. In contrast, it should have been particularly easy to assign the correct orientation to axisextended pictures, since the main axis of elongation of such pictures coincided with and emphasized the main axis of elongation of a typical picture of that object. This should have been especially helpful when misoriented, as compared with upright, views of axis-extended pictures were identified. On this account, axis-switched pictures should, therefore, have produced larger misorientation costs than did axis-extended pictures. The results of Experiment 3 did not, however, support this prediction.
There was an increased misorientation cost for axisswitched pictures relative to axis-normal pictures. However, the same increase in misorientation cost was found for axis-extended pictures, as compared with axis-normal pictures. In addition, there was the same increase in the difficulty of naming upright views of axis-switched and axis-extended pictures relative to the naming of axisnormal pictures. Overall, the naming of both upright and misoriented views of axis-switched and axis-extended pictures was very similar and was significantly worse than that for axis-normal pictures. Both axis switching and axis extension degraded and distorted the axis-normal pictures. This increased difficulty in identifying the axismanipulated stimuli interacted with the effects of plane 
misorientation to increase the misorientation cost (see also Lawson \& Jolicœur, 1998, 2003).

\section{EXPERIMENT 4}

The results of Experiments 2 and 3 suggest that the ease of specifying the main axis of elongation of an object does not play a specific role in the identification of misoriented views of that object. There is, though, an alternative explanation of these results, which was tested in Experiment 4. In Experiments 2 and 3, half the stimuli were seen at misoriented views. Since such an unusually high proportion of stimuli were misoriented in these studies, the participants may not have assumed by default that the stimuli were upright. Instead, they may have tried to determine the orientation of all the stimuli presented. This would have been especially likely for the distorted (axis-extended and axis-switched) pictures, where even upright views would not have matched well to the stored, canonical representations of upright objects.

If it was important to determine the orientation of all the stimuli, both upright and misoriented axis-extended pictures could have benefited from having a main axis of elongation that was easier to specify, relative to axisswitched pictures. In this case, any differences in responding to axis-extended and axis-switched pictures would not be predicted to be revealed specifically for misoriented stimuli. Instead, axis manipulation effects should be similar for upright and for misoriented views, as was observed in Experiments 2 and 3. In both experiments, performance with both upright and misoriented axis-switched pictures may have been poor because the main axis of elongation was harder to specify, relative to axis-extended and axis-normal pictures. In contrast, performance with both upright and misoriented axis-extended pictures may have been poor because these depictions were of worse quality than those of the axis-switched and axis-normal pictures. These two effects may have roughly balanced each other out. If so, there would be equally poor overall performance for axis-switched and axis-extended pictures, relative to axis-normal pictures, as was observed in Experiments 2 and 3.

Experiment 4 tested this alternative account by having participants name only upright views of the axis-switched, axis-extended, and axis-normal pictures presented in Experiments 2 and 3. Since the participants in Experiment 4 saw no misoriented stimuli, they could adopt the default assumption that all the stimuli presented were shown upright. In contrast to Experiments 2 and 3, they were not encouraged to determine the orientation of any stimuli, since there was never a need for them to use orientationsensitive transformation processes. Instead, all the stimuli could be identified by being matched directly to stored upright representations.

On the alternative account given above, performance in Experiment 4 should be worse for the axis-extended pictures than for the axis-switched pictures, in contrast to Experiments 2 and 3, because problems in determining the main axis of elongation of the axis-switched pictures should not disrupt performance in Experiment 4, so axis-switched pictures should be relatively easy to name. In contrast, difficulties in identifying poor quality axis-extended pictures would be the same in Experiment 4 as in Experiments 2 and 3, so axis-extended pictures should remain hard to name.

If, in contrast, the results of Experiment 4 replicated those of Experiments 2 and 3, with upright views of axisswitched and axis-extended pictures being equally difficult to name, this would support the conclusion that specifying an object's main axis of elongation does not play any particular role in identifying misoriented views of that object. Evidence in support of the latter conclusion was provided by Experiment 1, in which only upright stimuli were presented. Here, there was no evidence that axisswitched pictures were easier to classify as wide or tall than axis-extended pictures. Furthermore, the results were very similar to those for upright views in Experiment 2 (in which misoriented, as well as upright, views were presented). However, the wide or tall task used in Experiments 1 and 2 only indirectly assessed object identification. In contrast, Experiment 4, like Experiment 3, used a naming task that directly measured object identification.

\section{Method}

Participants. Eighteen undergraduate students at the University of Liverpool volunteered to take part in the study for course credit.

Materials. The upright views of the axis-normal, axis-switched, and axis-extended pictures of the 16 Tall-A, 16 Tall-B, 16 Wide-A, and 16 Wide-B objects presented in Experiment 1 were used here.

Design. The participants completed one experimental block of 192 naming trials. In this block, each of the 64 objects was seen once as an axis-normal, axis-switched, and axis-extended picture. The block comprised three subblocks, with a self-timed rest period between each. The participants named each object once in every subblock, and there were approximately equal numbers of axisnormal, axis-switched, and axis-extended pictures in each subblock. The order of presentation of subblocks was counterbalanced in a Latin square design. Three subgroups of 6 participants were assigned to each of the three subblock orders. Prior to the first block of naming, the participants completed a block of 16 practice trials. These were identical to experimental trials, except that no axis-normal pictures were presented; instead, a mixture of upright axis-extended and axis-switched pictures were shown to increase participants' exposure to these distortions. No objects seen in practice were included in the experimental trials.

Apparatus and Procedure. The apparatus and procedure were identical to those in Experiment 3.

\section{Results}

Trials in which the participants used an inappropriate name or in which the microphone was accidentally activated before the participant had responded were discarded as errors. In addition, RTs less than $500 \mathrm{msec}$ or exceeding $2,500 \mathrm{msec}$ were discarded as errors (less than $2 \%$ of the trials). The participants were replaced if they scored over $20 \%$ errors. Two participants were replaced using this criterion. There was one empty cell in the 
items analysis of RTs, which was filled by the mean for that condition. ANOVAs were conducted on the mean correct RTs and the percentages of errors.

There were three within-subjectsfactors, type-distortion (axis normal, axis extended, or axis switched), 3-D object axis (wide or tall), and stimulus set (if Set A was presented unaltered and Set B distorted or vice versa), and one between-subjects factor, condition (the order of presentation of subblocks; ABC, BCA, or CAB). The 3-D object axis, stimulus set, and condition counterbalancing factors were included in the analyses, but effects involving these factors were not the focus of interest, and so, for clarity of presentation, they are not reported here. All comparisons noted are significant at $p<.05$ in post hoc Newman-Keuls analyses.

Naming upright views. Type-distortion was significant for RTs $\left[F_{\mathrm{p}}(2,30)=74.713, p<.001 ; F_{\mathrm{i}}(2,120)=\right.$ $15.268, p<.001$; see Figure 5] but not errors $\left[F_{\mathrm{p}}(2,30)=\right.$ $\left.2.426, p>.1 ; F_{\mathrm{i}}(2,120)=2.443, p>.09\right]$ ]. Axisswitched pictures (924 msec, $9.7 \%$ ) and axis-extended pictures (937 $\mathrm{msec}, 7.8 \%$ ) were named more slowly than axis-normal pictures ( $852 \mathrm{msec}, 7.7 \%$ ). Most important, there was no indication that axis-extended pictures were harder to name than axis-switched pictures.

\section{Discussion}

The results of Experiment 4 replicated those of Experiment 3 in showing that upright views of axis-switched and axis-extended pictures were equally difficult to name, relative to axis-normal pictures. This result is consistent with the conclusion drawn from the results of Experiments 2 and 3 that specifying an object's main axis of elongation plays no particular role in identifying misoriented views of that object. There was no evidence for the alternative hypothesis that the results of Experiments 2 and 3 were due to the high proportion of misoriented stimuli presented

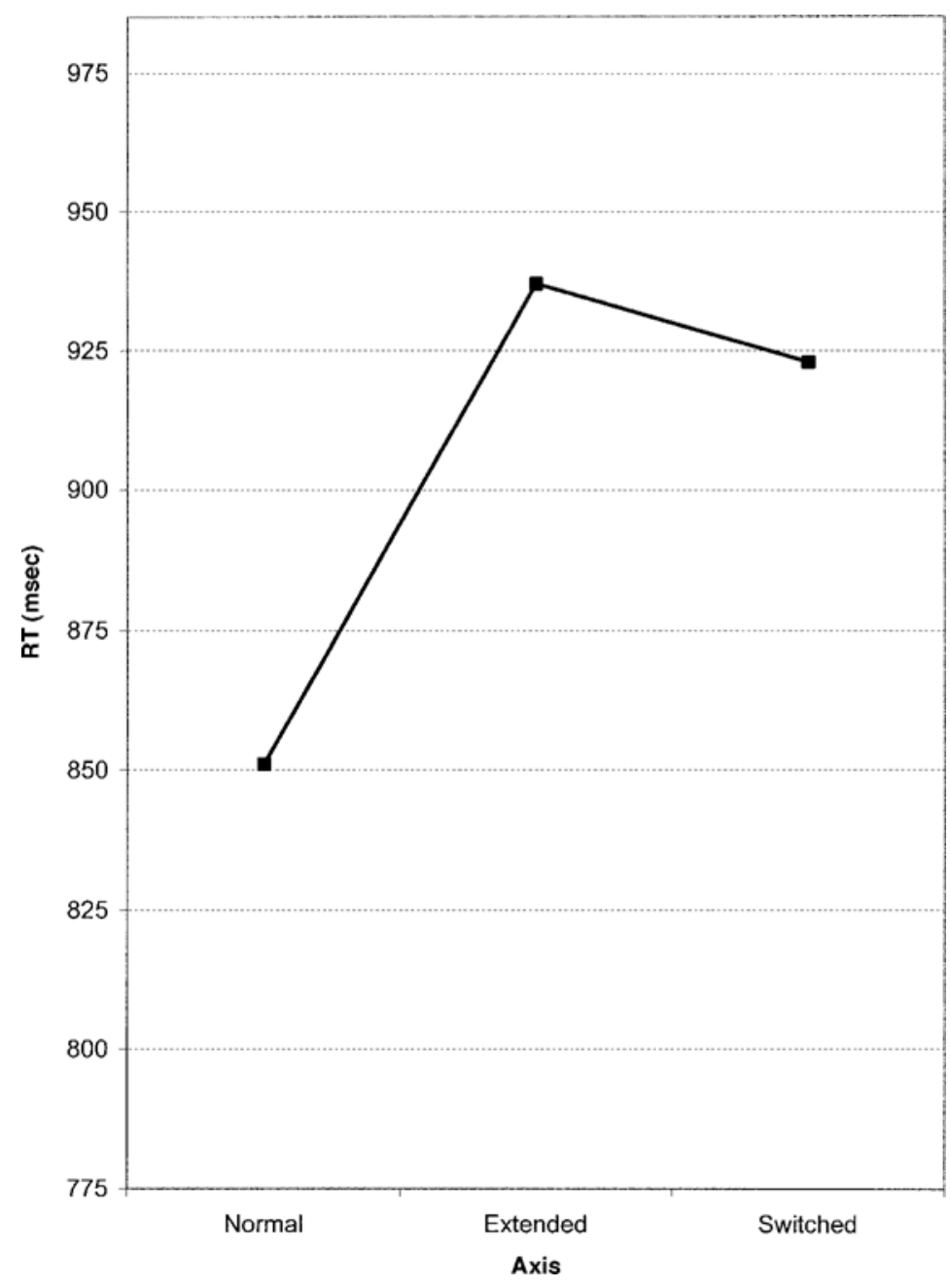

Figure 5. Mean reaction times (RTs) to name upright axis-normal, axisextended, and axis-switched pictures in Experiment 4. 
that encouraged the participants to try to identify the orientation of all the stimuli, upright as well as misoriented. The latter account predicted that axis-switched pictures would be easier to name than axis-extended pictures if only upright views were presented. Experiment 4 showed no evidence to support this prediction.

\section{GENERAL DISCUSSION}

A popular account of the identification of misoriented views of familiar objects proposes that when a misoriented view is first seen, its internal representation must be transformed to the upright before it can be matched to a stored upright object representation (Jolicœur, 1990). However, Corballis (1988) noted an apparent paradox in this hypothesis. How, without first identifying the stimulus, does the transformation process determine the likely orientation of that stimulus in order to appropriately transform it to the upright so that it can be identified? If the stimulus orientation is known before the stimulus has been transformed, it suggests that the stimulus has already been identified, in which case the transformation process is unnecessary.

The four experiments reported here tested one possible explanation of this paradox. If the visual system could use reliable cues to orientation that were not stimulus specific, such as axis elongation, it could determine the likely orientation of an object without first identifying the object. In four experiments, the importance of the depicted orientation and extent of the main axis of elongation of an object for classifying stimuli as wide or tall (in Experiments 1 and 2) and for identifying stimuli (in Experiments 3 and 4) for upright views only (in Experiments 1 and 4) and for both upright and plane-misoriented views (in Experiments 2 and 3) was examined.

The results from Experiments 1 and 2 confirmed that the axis manipulations used in all four experiments had a powerful effect on performance in a wide or tall? task in which people specified the orientation of the main axis of elongation of a stimulus. For axis-switched pictures, the main axis of elongation was depicted as perpendicular to the object's usual main axis (see Figure 1). In contrast, for axis-extended pictures, the depicted main axis of elongation was both highly salient and consistent with its usual orientation for that object. As a result, it was much harder to specify the orientation of an object's main axis of elongation for both upright and misoriented views of axis-switched pictures, relative to axis-extended pictures. However, although it was much harder to classify axis-switched pictures as wide or tall, as compared with axis-extended pictures, Experiments 3 and 4 showed no difference in the naming of either upright or misoriented views of axis-switched and axis-extended pictures.

Critically, in neither Experiment 2 nor Experiment 3 was the misorientation cost greater for axis-switched pictures (in which the position of the main axis of elongation of the picture conflicted with that of the real, 3-D object) than for axis-extended pictures (in which the main axis of elongation of the picture and of the object coincided, and the elongation of the main axis was accentuated). This indicates that the image transformation route to recognition is not influenced by the depicted position and extent of the main axis of elongation of the object.

Other accounts of the pattern of observed misorientation costs need to be tested. One possibility is that cues other than elongation (e.g., symmetry, generic shape) allow object orientation to be determined before misoriented views of objects are identified. An alternative explanation is that the orientation of misoriented stimuli is not known prior to image transformation. Instead, the visual system may try to transform an unidentified misoriented stimulus in multiple different ways simultaneously, with processing continuing until one of these parallel processing routes to identification succeeds in mapping the stimulus to a stored upright object representation. Note, though, that such an account is far removed from the typical assumption that a single incremental process, such as mental rotation, acts on a misoriented stimulus to normalize it to the upright by transforming it in one particular direction. Given that additional image transformations may be needed to identify any given stimulus (e.g., to compensate for depth rotation, scaling, occlusion, distortion, etc.), such an account suggests that compensating for plane misorientation would require considerable processing power.

\section{REFERENCES}

Corballis, M. C. (1988). Recognition of misoriented shapes. Psychological Review, 95, 115-123.

Corballis, M. C., Zbrodoff, N. J., Shetzer, L. I., \& Butler, P. B. (1978). Decisions about identity and orientation of rotated letters and digits. Memory \& Cognition, 6, 98-107.

DE CARO, S. A. (1998). On the perception of objects and their orientations. Spatial Vision, 11, 385-400.

DE CARo, S. A., \& REEves, A. (2000). Rotating objects to determine orientation, not identity: Evidence from a backward-masking/dualtask procedure. Perception \& Psychophysics, 62, 1356-1366.

De CARO, S. A., \& ReEves, A. (2002). The use of word-picture verification to study entry-level object recognition: Further support for view-invariant mechanisms. Memory \& Cognition, 30, 811-821.

Dell'ACQUA, R., \& JoB, R. (1998). Is object recognition automatic? Psychonomic Bulletin \& Review, 5, 496-503.

Dell'ACQuA, R., Job, R., \& GRAINGER, J. (2001). Is global shape sufficient for automatic object identification? Visual Cognition, 8, 801-821.

Gauthier, I., \& TARr, M. J. (1997). Orientation priming of novel shapes in the context of viewpoint-dependent recognition. Perception, 26, 51-73.

HAMM, J. P., \& MCMullen, P. A. (1998). Effects of orientation on the identification of rotated objects depend on the level of identity. Journal of Experimental Psychology: Human Perception \& Performance, 24, 413-426.

JOLICÄUR, P. (1985). The time to name disoriented natural objects. Memory \& Cognition, 13, 289-303.

JOLICÄUR, P. (1990). Identification of misoriented objects: A dual systems theory. Mind \& Language, 5, 387-410.

JolicÄUr, P., Corballis, M. C., \& LAwson, R. (1998). The influence of perceived rotary motion on the recognition of rotated objects. Psychonomic Bulletin \& Review, 5, 140-146.

JOLICÄUR, P., \& MiLlikEN, B. (1989). Identification of disoriented ob- 
jects: Effects of context of prior presentation. Journal of Experimental Psychology: Learning, Memory, \& Cognition, 15, 200-210.

LAWSON, R. (1999). Achieving visual object constancy across plane rotation and depth rotation. Acta Psychologica, 102, 221-245.

LAWSON, R., \& JOLICÄUR, P. (1998). The effects of plane rotation on the recognition of brief masked pictures of familiar objects. Memory \& Cognition, 26, 791-803.

LAWSON, R., \& JOLICÄUR, P. (1999). The effect of prior experience on recognition thresholds for plane-disoriented pictures of familiar objects. Memory \& Cognition, 27, 751-758.

LAWSON, R., \& JOLICÄUR, P. (2003). Effects of visual similarity on recognition thresholds for plane-misoriented pictures of familiar objects. Acta Psychologica, 112, 17-41.

MARR, D. (1982). Vision. San Francisco: Freeman.

McMullen, P. A., Hamm, J., \& JolicÄUR, P. (1995). Rotated object identification with and without orientation cues. Canadian Journal of Experimental Psychology, 49, 133-149.

MURRAY, J. E. (1998). Is entry-level recognition viewpoint invariant or viewpoint dependent? Psychonomic Bulletin \& Review, 5, 300-304.

SANOCKI, T. (1999). Constructing structural descriptions. Visual Cognition, 6, 299-318.

SEKuler, A. B., \& Swimmer, M. B. (2000). Interactions between symmetry and elongation in determining reference frames for object perception. Canadian Journal of Experimental Psychology, 54, 42-55.

SNODgrass, J. G., \& VANDERWART, M. (1980). A standardized set of 260 pictures: Norms for name agreement, image agreement, familiarity and visual complexity. Journal of Experimental Psychology: Human Perception \& Performance, 6, 174-215.

\section{NOTES}

1. The main axis of elongation of an object (e.g., horizontal for a bicycle) need not be the same as the main axis of elongation of some views of that object (a bicycle seen from the front or rear is mainly vertically elongated). I tried to avoid this complication by using stimuli for which, if the main axis of elongation of an object was horizontal (or vertical), the main axis of elongation of the axis-normal, upright picture of that object was also horizontal (or vertical). Due to a programming error, one exception to this was the camel. Pictures of two different exemplars of the camel were included in the experiment, one in the Wide-A set and one in the Tall-B set. The Tall-B set camel should not have been included in the experiment, since in most postures, camels are horizontally, rather than vertically, elongated. Nevertheless, since the data for both sets of camel pictures were similar to the results for other items in their respective conditions, I included the data in the analyses reported here.

2. For some of the axis-normal pictures (mainly tall stimuli with a large aspect ratio), an axis-switched picture that reversed the height-to-width aspect ratio would have so altered and degraded the picture that it would have been extremely hard to recognize. To avoid this, the imaginary box enclosing such pictures had a less extreme aspect ratio than the picture itself. For example, for a tall object, the box surrounding the axis-normal picture was somewhat wider than was necessary to just enclose it.

(Manuscript received November 12, 2002; revision accepted for publication June 25, 2003.) 